\title{
Towards joint optimization of product design, process planning and production planning in multi-product assembly
}

\author{
Daisuke Tsutsumi (3)a, Dávid Gyulaib,c, András Kovács ${ }^{b}$, Bence Tiparyb,c, Yumiko Ueno (3)a , Youichi Nonaka (3)a, \\ László Monostori (1) ${ }^{\mathrm{b}, \mathrm{c}}$ \\ a Hitachi Ltd. Research \& Development Group, Japan \\ ${ }^{b}$ Centre of Excellence in Production Informatics and Control, Institute for Computer Science and Control, Hungarian Academy of Sciences, Budapest, Hungary \\ ${ }^{c}$ Department of Manufacturing Science and Engineering, Budapest University of Technology and Economics, Budapest, Hungary
}

Offering product variety is crucial for satisfying diverse customer needs. Although product design, process and production planning related decisions are interdependent, they are conventionally made by different divisions, separately for each product, resulting in excess costs. This paper proposes a methodology for increasing investment efficiency by the joint optimization of product design, process and production planning for a family of products. Tolerance allocation, as a sub-problem of product design, and assembly resource configuration, regarding process planning, are solved jointly, with a foresight on long-term production planning. The efficiency of the method is demonstrated through an industrial case study.

Assembly, Tolerancing, Design optimization, Production planning

\section{Introduction and motivation}

Ever-diversifying consumer requirements force companies to increase product variety in many industries, including automotive, machinery, electronics or consumer goods, which also increases the demand for multi-product assembly [1][2]. At the same time, the conventional product development process addresses product design, process and production planning in a decomposed, sequential way [3], without appropriate feedback mechanisms, which often results in product designs that prevent the full exploitation of the synergies stemming from a common assembly system [4]. This, in turn, can easily lead to excessive investment costs and low Return on Asset (RoA) [5].

The objective of the research presented in the paper was to open new avenues from production back to design, so as to facilitate the most efficient use of existing assembly resources for new products. This paper proposes a methodology for increasing investment efficiency by the joint optimization of product design, process and production planning for a family of products.

In the product design stage, Design for Manufacturing and Assembly (DfMA) methodologies, first proposed by Boothroyd [6], provide guidelines and basic principles for developing product structure and geometry with the consideration of manufacturability and assemblability aspects. Various approaches to build such guidelines have been proposed for different branches of industry [7]-[9]. Recent research also addressed applying data mining techniques to organizing knowledge about assembly processes as a BOP (Bill of Process) and to utilize the BOP as rules for DfMA [10]. A major issue with conventional DfMA for multiproduct assembly is that too strict or inadequate guidelines may render it impossible to respond appropriately to customer needs, and hence, product designs violating the guidelines become inevitable. As a result, assembly equipment and systems need to deal with highly individualized products, resulting in excess investments, which implies that the efficiency of conventional DfMA decreases in multi-product assembly.
A key challenge in solving product design with an explicit consideration of process and production planning aspects is the inherent complexity of the latter problems. Process planning itself is typically solved by decomposition into sub-problems related to assembly sequence planning, assembly line balancing, and assembly path planning. A review of these sub-problems and the applicable solution approaches is provided in [11]. Furthermore, the automated generation of process plans requires well-defined formal data models capturing assembled products as well as assembly processes. Despite the ambitious efforts, such as the ontology model [12] of NIST, these are hardly used in practice. Instead, process planning methods are often specialized to certain families of products, lack any feedback mechanism to product design, and therefore, their efficiency in reducing equipment investment is insufficient.

As an initial but essential step towards the joint optimization of product design, process and production plans, the current paper focuses on tolerance allocation in product design, and assembly resource configuration in process and production planning. The products to be assembled are composed of multiple parts, and it is required to satisfy the tolerance specifications at minimum total cost, considering costs related to parts manufacturing, assembly, and equipment investment. There are two factors that may cause a violation of tolerance specifications: manufacturing deviations of individual parts and performance deviations of the assembly equipment; controlling either type of deviation is associated with a cost function [13][14]. Due to the fact that not only the costs, but also the quality of the products is strongly influenced by the tolerance scheme, tolerance allocation is one of the most important steps in product design development [13]. The location of tolerance allocation and assembly resource configuration in the overall product development process is shown in Fig. 1.

In the following sections, a detailed formulation of the problem and a proposed solution approach are presented along with experimental results on an industrial case study. 


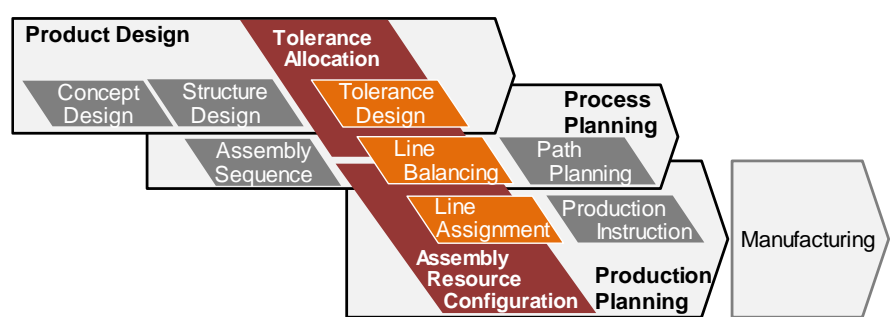

Fig. 1. Product development according to the proposed method.

\section{Problem statement}

\subsection{Overview}

The overall problem addresses finding the optimal tolerance allocation for a family of products that satisfies all design specifications and minimizes the total production cost, including part manufacturing, assembly, and potential investment costs. However, in order to achieve designs that can be manufactured and assembled efficiently, product design must be formed with a foresight on process planning and production planning. Accordingly, the problem has two main, interrelated sub-problems that must be solved jointly:

- The tolerance allocation sub-problem, which is responsible for selecting the structural design alternative to apply for each product (including potential adjustment mechanisms) and assigning tolerance values to individual dimensions in the most efficient way; and

- the assembly resource configuration sub-problem, which matches forecasted orders for the products to assembly equipment and configures the equipment to produce the selected design alternatives with the allocated tolerances. When performing this, both existing resources and the opportunity of investing into new resources are considered.

These definitions enable the solution of (a sub-problem of) product design with a solid foresight on process planning and production planning within the product development process presented in Fig. 1. Throughout the paper, it is assumed that new members of the product family use the existing production technology and partly the existing assembly resources, and hence, both the tolerances and the assembly resource configuration must be optimized for the given, fixed assembly sequence.

\subsection{Tolerance allocation sub-problem}

The tolerance allocation sub-problem is dedicated to finding such tolerance values for the dimensions in the dimension chains that result in feasible product geometry, functional product and minimal production costs. It is assumed that maintaining product functionality depends solely on satisfying the design specifications in terms of nominal and tolerance values of one or more main dimensions. Two possible approaches can be followed in order to meet the design specifications:

- Design specification can be reached by setting the tolerance values so that the stacked tolerance in the corresponding dimension chain is at most the specified tolerance value after manufacturing and assembly. In this case, no adjustment during assembly is possible by design; the specified dimension is driven by the geometry of the product, following the fully defined component connections.

- If the component connection is under-defined, the stacked tolerance of the dimension chain can be improved by adjustment during assembly. In this case, the precision of the adjustment process also has to be taken into account when allocating tolerances.

The objective is to select, for each product $p$, a structural design alternative $s$, from the set of structural alternatives provided in the input by a designer, and to allocate a tolerance value $\tau \delta$ to each individual dimension $\delta$ in such a way that the design specification (stacked tolerance, compensated by potential adjustment) $\varphi_{\Delta}$ is satisfied for each dimension chains $\Delta$ in $s$.

In case of dimension chains with adjustment, i.e., $\Delta \in \Delta_{s^{+}}$, the adjustment range $r_{\Delta}^{A}$ is also provided by the designer. For these chains, the required adjustment precision value $r_{\Delta}{ }^{B}$ must be specified, too, and the evaluation of stacked tolerance values has to be performed accordingly: the stacked tolerance is decreased with the adjustment range and increased with the adjustment precision (assuming that adjustment takes place after assembling all components).

In addition to satisfying design specifications, tolerance allocation needs to result in an optimal solution in terms of manufacturing costs as well. The total parts manufacturing costs are composed of a fixed base manufacturing $\operatorname{cost} C_{s}^{T 0}$ and a variant cost that depends on the allocated tolerance values for each individual dimension. The cost of manufacturing a dimension $\delta$ of the product to the selected tolerance $\tau \delta$ is approximated by a convex piecewise linear cost function, specified by a list of breakpoints $\left(\left(C_{1}{ }^{T[x]}, C_{1}^{T[y]}\right),\left(C_{2}^{T[x]}, C_{2}^{T[y]}\right), \ldots,\left(C_{R}^{T[x]}, C_{R}^{T[y]}\right)\right)$, where $C_{r}^{T[x]}$ represents a tolerance value and $C_{r}^{T[y]}$ stands for the corresponding manufacturing cost. From these data, the tolerance cost $C_{\delta}^{T}\left(\tau_{\delta}\right)$ can be calculated using the following formula:

$$
C_{\delta}^{T}\left(\tau_{\delta}\right)=\max _{r \geq 2}\left(C_{r-1}^{T[y]} \frac{C_{r}^{T[x]}-\tau_{\delta}}{C_{r}^{T[x]}-C_{r-1}^{T[x]}}+C_{r}^{T[y]} \frac{\tau_{\delta}-C_{r-1}^{T[x]}}{C_{r}^{T[x]}-C_{r-1}^{T[x]}}\right)
$$

\subsection{Assembly resource configuration sub-problem}

The assembly resource configuration sub-problem is responsible for assessing how the different design variants can be produced most efficiently on existing assembly lines considering capacity and capability constraints, or alternatively, what investments should be made into new assembly lines or into extending the capabilities of existing lines on a finite time horizon. Since all products are assembled in the same, common assembly system, the assembly resource configuration problems related to different products are interrelated.

Formally, there is given a set of design alternatives for each product. For running products, this set is singleton, and contains only the currently produced design, whereas for new products, there are multiple candidate design alternatives available. The selected design alternatives must be assembled on multi-product lines in a common assembly system. In each time period $t$, where there is nonzero demand $g_{p}{ }^{t}$ for a product $p$, the selected design alternative of $p$ must be assigned to exactly one assembly line, which can be either an existing line or a newly built one. While existing lines are available from the beginning of the time horizon, new lines can be built in any chosen time period, for a given investment $c^{L}$. The capacity of each existing and potential new line $l$ is fixed, $q_{l}$.

Design alternatives with adjustment mechanisms require specialized measurement and assembly equipment, and the alternative designs of a product may differ in the required equipment. These crucial capabilities are encoded in the form of some numerical parameters that characterize each alternative. For the sake of brevity, the current paper assumes one such crucial capability, the adjustment precision of the line, though the approach can be generalized to any finite number of those capabilities. The initial adjustment precision of line $l$ is denoted by $b_{l 0}$, which can be improved to $b_{l t}$ with $b_{l 0} \geq b_{l t} \geq \underline{b}$. Then, a given design alternative $d$ can be assembled on line $l$ if $r_{d}^{B}=\min _{\Delta \in d} r_{\Delta}^{B} \geq$ $b_{l t}$. Similarly to the parts manufacturing cost that depends on the allocated tolerance, the cost of the necessary equipment, as a function of the adjustment precision $b_{l t}$, is approximated by a 
convex piecewise linear cost function specified by a list of breakpoints $\left(\left(C_{1}^{P[x]}, C_{1} P[y]\right),\left(C_{2}^{P[x]}, C_{2} P[y]\right), \ldots,\left(C_{R}^{P[x]}, C_{R} P[y]\right)\right)$. From these values, the equipment $\operatorname{cost} C_{l}{ }^{P}\left(b_{l t}\right)$ can be calculated using a formula analogous to (1). Then, the investment related to improving the adjustment precision of line $l$ from $b_{l 0}$ to $b_{l t}$ equals to the required increase in equipment value $C_{l} P\left(b_{l t}\right)-C_{l} P\left(b_{l 0}\right)$. A linear depreciation model with a useful life of $T^{D}$ and zero salvage value is applied to investments, related both to building new lines and to extending the capabilities of existing or new ones. In order to ensure a linear depreciation throughout the planning horizon, this paper assumes that $T^{D}$ is greater than or equal to the length of the horizon.

It is allowed to reassign a product from one line to another, but this is penalized with a changeover cost of $c^{X}$ and a changeover time of $a^{X}$. Design alternatives also differ in the manufacturing cost of the parts $c d^{M}$ (determined by the allocated tolerances) and processing time on the assembly lines $a_{d}$ (input parameter). The cost of operating assembly line $l$ for a unit time equals $C_{l}{ }^{O}$, which covers labor costs, but does not include, e.g., electricity fees or line maintenance costs. It is assumed that there is no cost differentiation based on operator skills. The total cost of a solution is then composed of the parts manufacturing cost, the assembly lines operation cost, the changeover cost, as well as the investment costs related to new line installation and adjustment precision extension. It is assumed that these investment costs include not only the installation of equipment, but also the costs required for preparing the related operation methods. A solution minimizing the total cost is sought.

\section{Mathematical model}

\subsection{Decomposition approach}

The tolerance allocation and the assembly resource configuration sub-problems are inherently interconnected: while the tolerance allocation sub-problem defines alternative designs for the products, the costs of producing the different designsconsidering the currently available and potential future assembly equipment-can be assessed only during assembly resource configuration. This section proposes a decomposition scheme that allows jointly solving the two sub-problems in such a way that the resulting solution is globally optimal for the overall problem.

The decomposition approach consists of solving first multiple instances of the tolerance allocation sub-problem to generate a portfolio of design alternatives for each product; then, assembly resource configuration selects a design alternative for each product and matches it to assembly resources in the most efficient way. Hence, the assembly resource configuration sub-problem is solved only once, for the ensemble of all products and all (currently existing and potential new) assembly lines. The decomposition scheme is depicted in Fig. 2.

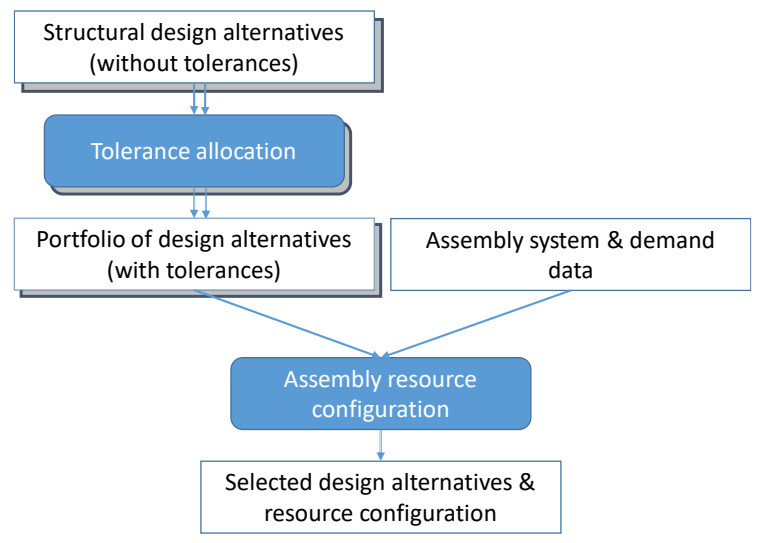

Fig. 2. Proposed decomposition scheme.
Since the tolerance allocation sub-problem is a continuous problem, it may admit infinitely many feasible solutions for each product. For the decomposition scheme to ensure a globally optimal solution, it is therefore crucial to characterize design alternatives that can take part in optimal solutions, and to identify a finite portfolio of design alternatives that is guaranteed to contain an optimal design alternative for each product. The key to achieving this is exploiting the linearity of the constraints in the problem and the piecewise linearity of all relevant cost functions, which implies that at least one of the optimal solutions lies in either at the bounds of the feasible interval or at the breakpoints of those cost functions. Moreover, it is exploited that the design alternatives derived from the same structural design of a product, on the level of production costs and requirements captured by the assembly resource configuration model, differ solely in their adjustment precision requirement and their part manufacturing cost, whereas all other parameters (including capacity requirements, line operation costs, etc.) are common. Therefore, the portfolio can be generated by imposing different bounds on the adjustment precision variables, which result in different trade-offs between adjustment precision requirement and part manufacturing costs (i.e., mild precision requirements on the line but high parts manufacturing costs due to strict tolerances, or strict adjustment precision requirement but lower parts manufacturing costs).

The portfolio must, therefore, contain for each product and for each structural design alternative of the product the following tolerance design alternatives:

1. For each existing assembly line, the current adjustment precision $b_{l 0}$ of the line. For each potential newly constructed line, the initial adjustment precision of the line, assuming that the line is constructed.

2. The breakpoints $C_{r}^{P[x]}$, for $r=1, \ldots, R$ of the adjustment precision cost function of the assembly lines.

3. Values $\varphi_{\Delta}+r \Delta^{A}-/ \Delta / C_{r} T[x]$ for $r=1, \ldots, R$ for all dimension chains with adjustment in all products, where $/ \Delta /$ denotes the number of dimensions in the dimension chain (excluding the adjusted dimension), provided that they fall into the feasible interval $\left[C_{1} P[x], C_{R}^{P[x]}\right]$ for adjustment precisions. These values induce a breakpoint in the tolerance cost functions (refer to constraint (7) in the tolerance allocation model).

It is noted that this paper assumes a uniform tolerance cost function for all dimensions. With different costs functions for individual dimensions, the definition given in point 3 above can be trivially adjusted.

\subsection{Tolerance allocation sub-problem}

The tolerance allocation sub-problem for a given structural alternative $s$ of a product and a given adjustment precision value $r_{d}^{B}$ can be represented as a linear program (LP) as follows:

minimize

$\sum_{\delta} C_{\delta}^{T}$

subject to

$\sum_{\delta \in \Lambda} \tau_{\delta} \leq \varphi_{\Delta}$

$\forall \Delta \in \Delta_{s}$

$\sum_{\delta \in \Delta} \tau_{\delta}-r_{\Delta}^{A}+r_{\Delta}^{B} \leq \varphi_{\Delta}$

$\forall \Delta \in \Delta_{s^{+}}$

$r_{d}^{B} \leq r_{\Delta}^{B} \leq \varphi_{\Delta}$

$\forall \Delta \in \Delta_{s}^{+}$

$C_{1}^{T[x]} \leq \tau_{\delta} \leq C_{R}^{T[x]}$

$\forall \delta$

$C_{\delta}^{T} \geq\left(C_{r-1}^{T[y]} \frac{C_{r}^{T[x]}-\tau_{\delta}}{C_{r}^{T[x]}-C_{r-1}^{T[x]}}+C_{r}^{T[y]} \frac{\tau_{\delta}-C_{r-1}^{T[x]}}{C_{r}^{T[x]}-C_{r-1}^{T[x]}}\right) \quad \forall \delta, r \geq 2$ 
The objective is minimizing the total tolerance cost (2), composed of the costs of manufacturing the individual dimensions to the selected tolerances. Constraint (3) requires that the stacked tolerance along any dimension chain without adjustment is at most the design specification for the given chain. In contrast, for chains with adjustment, the adjustment mechanism can compensate an error equal to the adjustment range of the mechanism minus its adjustment precision (4). At the same time, the adjustment precision itself cannot be looser than the tolerance specification of the chain, or stricter than the precision limit set for the current instance (5). Bounds for the individual tolerances must be in line with technological limits (6), and the cost related to the tolerance on an individual dimension is determined by the piecewise linear cost function $C_{\delta}^{T}\left(\tau_{\delta}\right)$ (7).

\subsection{Assembly resource configuration sub-problem}

The assembly resource configuration sub-problem for the ensemble of all products has been encoded in the form of the following mixed-integer linear program (MILP) over binary decision variables $x$ dit to indicate that design alternative $d$ is assigned to line $l$ in period $t ; y_{d}$ to denote that design alternative $d$ is selected for production; $u_{d l t}$ to show that design alternative $d$ is reassigned to line $l$ in period $t$ from some other line; and $z l t$ to indicate that new line $l$ is constructed in period $t$. Continuous decision variables $b_{l t}$ represent the adjustment precision of line $l$ in period $t$, whereas $C_{l t}{ }^{P}$ stands for the cost of line $l$ as of period $t$ required for achieving the above precision. Auxiliary variables, used for improving the readability of the MILP (otherwise, they could be moved to the objective), include the parts' manufacturing cost $C^{M}$, the assembly line operation cost $C^{L}$, the changeover cost $C^{X}$, the new line installation cost $C^{I}$, and the assembly lines' adjustment precision extension costs $C^{P}$. The sum of these five cost components constitute the optimization criterion (8) of this MILP:

$$
\begin{array}{ll}
\text { minimize } & \\
C^{M}+C^{L}+C^{X}+C^{I}+C^{P} & \\
\text { subject to } & \forall p \\
\sum_{d \mid p(d)=p} y_{d}=1 & \forall d, t: g_{p(d) t)}>0 \\
\sum_{l} x_{d l t}=y_{d} & \forall d, l \in L^{n e w}, t \\
x_{d l t} \leq z_{l t} & \forall d, l, t \\
r_{d}^{B}-b_{l t} \geq(\bar{b}-\underline{b})\left(1-x_{d l t}\right) & \forall d, l, t \\
u_{d l t} \geq x_{d l t}-x_{d l(t-1)} & \forall l, t \\
\sum_{d}\left(g_{p(d) t} a_{d} x_{d l t}+a^{X} u_{d l t}\right) \leq q_{l} & \forall l \in L^{n e w}, t \\
z_{l t} \leq z_{l(t-1)} & \forall l, t \\
\underline{b} \leq b_{l t} \leq b_{l(t-1)} & \forall l, t, r \geq 2 \\
C_{l t}^{P} \geq\left(C_{r-1}^{P[y]} \frac{C_{r}^{P[x]}-b_{l t}}{C_{r}^{P[x]}-C_{r-1}^{P[x]}}+C_{r}^{P[y]} \frac{b_{l t}-C_{r-1}^{P[x]}}{C_{r}^{P[x]}-C_{r-1}^{P[x]}}\right) & \\
C^{P}=\sum_{l t} \frac{1}{T^{D}}\left(C_{l t}^{P}-C_{l 0}^{P}\right) & \\
C^{M}=\sum_{d l t}\left(C_{d}^{M} g_{p(d) t} x_{d l t}\right) & \\
C^{L}=\sum_{d l t}\left(c_{l}^{O} g_{p(d) t} a_{d} x_{d l t}\right) & \\
C^{X}=\sum_{d l t} c^{X} u_{d l t} & \\
C^{I}=\sum_{l t} \frac{c^{L}}{T^{D}} z_{l t} & \\
x_{d l t}, y_{d}, u_{d l t}, z_{l t} \in\{0,1\} &
\end{array}
$$

$b_{l t} \geq 0$

$\forall d, l, t$

Constraint (9) states that for each product, exactly one design alternative must be selected for production. Equation (10) ensures that the selected design alternatives are assigned to exactly one assembly line in each time period where there is nonzero demand for the given product. Products can be assigned to new lines only if they are installed (11), and to any line if the adjustment precision of the line is at least as good as the precision required by the design alternative (12). Here, $\bar{b}$ and $\underline{b}$ are the poorest and best possible adjustment precision of the lines, respectively. Constraint (13) relates the changeover variables to the assignment variables. The capacity constraint (14) states that the sum of processing times and changeover times on an assembly line, either existing or newly built, cannot exceed the line capacity. Investments related to new line installation (15) and adjustment precision extension (16) are performed in a given period of time, and they cannot be undone later. Inequality (17) calculates the adjustment precision costs of the individual lines in each time period. From these values, the total adjustment precision extension cost is computed using equality (18), by subtracting the cost of the initial lines from the extended lines, also accounting for depreciation. Then, equations (19-22) calculate the manufacturing, the line operation, the changeover, as well as the new line installation costs, respectively. Finally, constraints (23) and (24) define the variables as binary or non-negative continuous.

\section{Industrial case study}

\subsection{Production environment and experimental scenarios}

In this case study, the optimal tolerance design of mechanical assemblies consisting of multiple parts is investigated. Product design input data includes the structural design alternatives for each product, specifying the nominal geometry, the possibility of mechanical adjustment, as well as draft tolerances assigned by a human designer, which are used as reference values in the case study. The product family contains eight different products, three of which are new. The tolerance allocation problem is solved only for the new products, whereas the design of existing products cannot be modified. Each new product has two structural design alternatives, one with, and one without adjustment mechanism. The complete assembly procedure, in reality, consists of five processes, but for the sake of clarity, only the single critical process is considered in this study. This assembly process relates to a single dimension chain with at most one mechanically adjustable dimension. A structural design alternative with adjustment mechanism for a sample product and the investigated dimension chain are shown in Fig. 3. The design specifications differ for each product. A piecewise linear tolerance cost function is also provided for every dimension, specifying the manufacturing cost as a function of the allocated tolerance.

The input data required for the assessment of the incurred production costs contains the specifications of assembly lines (together with the investment opportunities into new lines or into
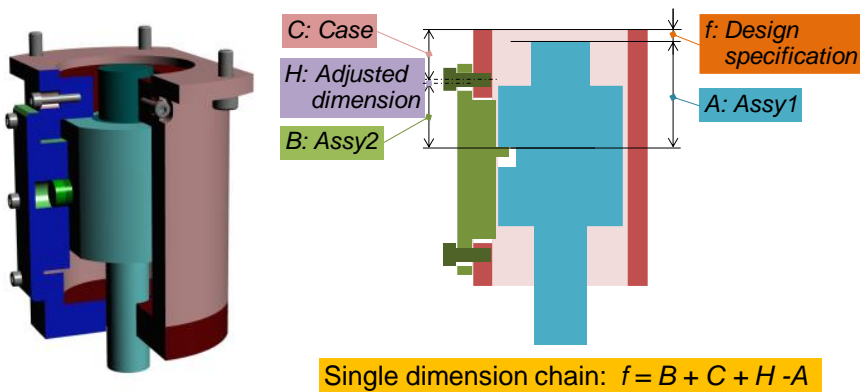

Fig. 3. Sample product. 
Table 1. Overview of the scenarios investigated in the case study.

\begin{tabular}{|l|c|c|c|}
\hline & $\begin{array}{c}\text { (A) } \\
\text { Draft }\end{array}$ & $\begin{array}{c}\text { (B) } \\
\text { Opt. tol. }\end{array}$ & $\begin{array}{c}\text { (C) } \\
\text { Opt. tol. \& adj. }\end{array}$ \\
\hline (1) Without adjustment & \#A1 & \#B1 & - \\
\hline (2) With adjustment & \#A2 & \#B2 & \#C2 \\
\hline (3) Mix by a human designer & \#A3 & \#B3 & \#C3 \\
\hline (4) Selection from all alternatives & \#A4 & \#B4 & \#C4 \\
\hline
\end{tabular}

extended capabilities), as well as product demand data. The time horizon is divided into 15 periods. In the beginning of the time horizon, three assembly lines exist, one of which has equipment for adjustment with a given initial precision. Reassigning a product from one line to another is penalized with a changeover cost and a changeover time equal to $1 / 30$ period. Line capacities are such that it is necessary to construct one or two new lines in addition to the existing lines. For all investments, a linear depreciation model with a 16-period useful life and zero salvage value is applied. Production volumes are given based on forecasts.

The objective of this case study is the assessment of the efficiency of the proposed approach in reducing total production costs, with special attention to the change in the balance between manufacturing cost for more precise parts and investment cost associated to assembling parts with a higher adjustment precision.

The scenarios analyzed in the case study are characterized in Table 1. Three different options have been investigated for solving the tolerance allocation sub-problem, corresponding to the columns of the table, which were implemented by fixing some of the decision variables of the proposed LP where necessary. The baseline is using the draft tolerances and adjustment precisions provided by a designer (A); the optimization of the part tolerances while still using the draft adjustment precisions (B); and the optimization of both part tolerances and adjustment precision requirements (C). Additionally, four cases are differentiated based on the availability of structural design alternatives for the three new products, corresponding to the rows of the table: only the alternatives without adjustment are considered (1); only with adjustment (2); without adjustment for one product and with adjustment for two products, which was considered to be the optimal mix by the human designer (3); and all structural alternatives can be selected (4). Scenario \#C1 does not exist, since no adjustment precision can be assigned to an alternative without adjustment. This results in a total of eleven scenarios investigated.

It is noted that in scenarios where a structural alternative with adjustment is present (2-4), the proposed approach computes a portfolio of toleranced design alternatives from which a selection is made during assembly resource configuration. In this case study, this resulted in 3-7 toleranced design alternatives for each such structural alternative.

\subsection{Analysis of the results}

The proposed approach has been implemented in the FICO Xpress mathematical programming suite, and it has been applied to solving the above defined eleven scenarios to exact optimality. The results are shown in a diagram in Fig. 4, where the vertical axis corresponds to the manufacturing and operation cost, whereas the horizontal axis stands for the investment cost. Since the total production cost is the sum of these two components, the diagonal lines represent solutions with equal total cost. The cost structure of the solutions is presented in detail in Table 2.

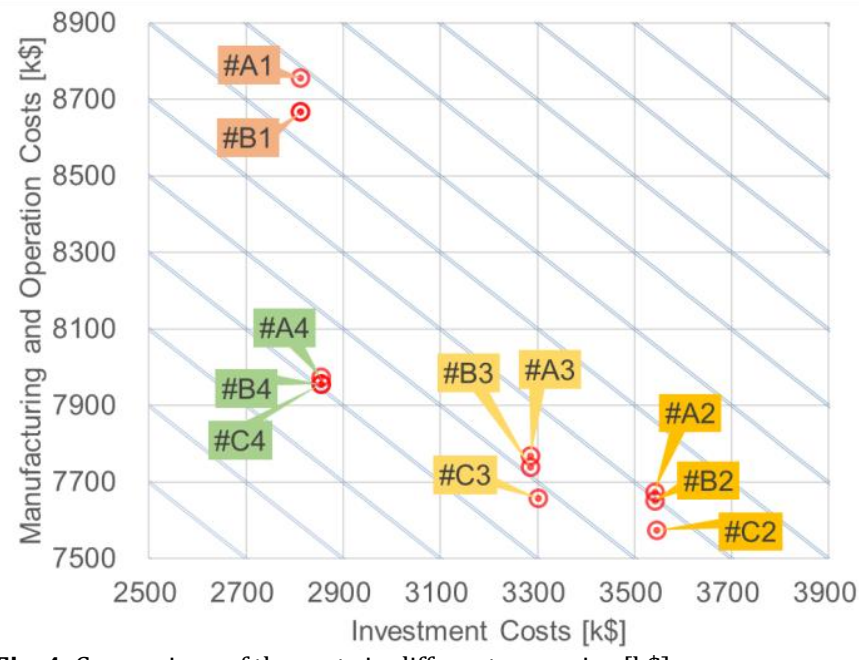

Fig. 4. Comparison of the costs in different scenarios $[\mathrm{k} \$]$.

Regarding the impact of tolerance allocation, optimized tolerances (B) resulted in a reduction of the total production cost by $0.1-0.8 \%$ compared to the tolerances specified by the human designer (A) for any combination of structural design alternatives (e.g., \#A1 to \#B1: 0.8\% reduction). This reduction occurred completely in the parts manufacturing costs. When optimizing the tolerances and adjustment precisions together (C), the reduction of the total cost reached up to $0.9 \%$. This means that the human designer performed well in solving the tolerancing problem alone.

Considering the differences stemming from the diverse structural alternatives available, the investment cost is the lowest in scenarios \#A1 and \#B1, where the alternatives without adjustment put minimal requirements on the assembly equipment. However, the manufacturing cost is the highest in these scenarios. The case is just the opposite when adjustment is required for all the three products (\#A2, \#B2, \#C2). The mix of structural alternatives composed by the human designer (\#A3, \#B3, \#C3) could indeed find a better compromise between manufacturing and investment costs.

Table 2. Cost structure of the optimal solutions under different scenarios [k\$].

\begin{tabular}{|c|c|c|c|c|c|c|c|c|c|c|c|}
\hline Scenario & \#A1 & \#A2 & \#A3 & \#A4 & \#B1 & \#B2 & \#B3 & \#B4 & $\# \mathrm{C2}$ & \#C3 & \#C4 \\
\hline Total production cost [\%]: & 100.0 & 96.9 & 95.6 & 93.6 & 99.2 & 96.8 & 95.3 & 93.4 & 96.1 & 94.7 & 93.4 \\
\hline Total production cost: & 11569 & 11216 & 11055 & 10829 & 11481 & 11193 & 11025 & 10810 & 11119 & 10960 & 10810 \\
\hline Operation cost $\left(c^{L}\right)$ : & 180 & 180 & 180 & 180 & 180 & 180 & 180 & 180 & 180 & 180 & 180 \\
\hline Changeover cost $\left(c^{X}\right)$ : & 6 & 8 & 9 & 6 & 6 & 8 & 9 & 6 & 8 & 9 & 6 \\
\hline Manufacturing cost $\left(c^{M}\right)$ : & 8571 & 7486 & 7579 & 7789 & 8482 & 7464 & 7550 & 7770 & 7387 & 7469 & 7770 \\
\hline Precision extension cost $\left(c^{P}\right)$ : & & 729 & 786 & 42 & & 729 & 786 & 42 & 732 & 802 & 42 \\
\hline Installation cost $\left(c^{l}\right)$ : & 2813 & 2813 & 2500 & 2813 & 2813 & 2813 & 2500 & 2813 & 2813 & 2500 & 2813 \\
\hline $\begin{array}{l}\text { Manufacturing and operation } \\
\left(c^{L}+c^{X}+c^{M}\right):\end{array}$ & 8757 & 7674 & 7768 & 7974 & 8668 & 7652 & 7739 & 7956 & 7574 & 7658 & 7956 \\
\hline Investment $\left(c^{P}+c^{I}\right)$ : & 2813 & 3542 & 3286 & 2854 & 2813 & 3542 & 3286 & 2854 & 3545 & 3302 & 2854 \\
\hline
\end{tabular}


Ultimately, the ability to select the appropriate combination of structural alternative together with optimal tolerance allocation resulted in a cost reduction of $6.6 \%$ from the baseline scenario \#A1 to the complete scenario \#C4, which is a considerable saving. For the particular problem instance, scenarios \#C4 and \#B4 led to identical results, because the draft adjustment precision was already optimal for the single product where a design alternative with adjustment was selected. With this, \#C4 (and \#B4) achieved the smallest total cost and found the best balance between manufacturing cost and investments among all scenarios. It should be noted that the selected structural alternatives were different from the mix composed by the human designer using a rule of thumb approach in scenarios (3). This proves that the proposed approach can provide an optimal combination of design alternatives and tolerance allocation to the designer.

Compared to earlier tolerance allocation models focusing on a single product with cost functions assigned to tolerances on individual dimensions [13][14][15], the benefit of the proposed approach is capturing the synergies of producing a family of products in a multi-product assembly system, and efficiently exploiting them to reduce the total production cost.

Table 3. Examples of tolerance allocation results [mm].

\begin{tabular}{|c|c|c|c|c|c|}
\hline \multirow{2}{*}{ Scenario } & \multirow{2}{*}{ Product } & \multicolumn{3}{|c|}{ Part tolerances } & \multirow{2}{*}{$\begin{array}{l}\text { Adjustment } \\
\text { precision }\end{array}$} \\
\hline & & A & B & C & \\
\hline \multirow{3}{*}{ \#A1 } & P1 & 0.2 & 0.2 & 0.1 & - \\
\hline & P2 & 0.1 & 0.1 & 0.05 & - \\
\hline & P3 & 0.04 & 0.04 & 0.02 & - \\
\hline \multirow{3}{*}{ \#C3 } & P1 & 0.2 & 0.25 & 0.05 & - \\
\hline & P2 & 0.25 & 0.25 & 0.15 & 0.1 \\
\hline & P3 & 0.2 & 0.25 & 0.05 & 0.1 \\
\hline \multirow{3}{*}{ \#C4 } & P1 & 0.2 & 0.25 & 0.05 & - \\
\hline & P2 & 0.1 & 0.1 & 0.05 & - \\
\hline & P3 & 0.2 & 0.25 & 0.05 & 0.1 \\
\hline
\end{tabular}

\section{Conclusion and future works}

In this research, an optimization approach was proposed, integrating product design, process planning, and production planning with the aim of improving RoA for a family of products. As a first step of the research, the paper focused on jointly solving tolerance allocation, as a sub-problem of product design, and assembly resource configuration, regarding process and production planning, in mechanical assembly. An industrial case study demonstrated the effectiveness of the joint optimization method and confirmed that it finds the optimal balance between the costs of manufacturing parts to the desired tolerances, and the investment costs related to the precision improvement of the assembly equipment. The approach provides an environment for the product designer and the production engineer where the required changes in product design and investments into assembly equipment can be determined and analysed integrally.

Future works will focus on extending the optimization model by considering more detailed production planning decisions. Examples of possible refinements include the option of overtime or investment into individual assembly resources. In a more ambitious research step, the product design component of the approach will be extended from tolerance allocation to proposing changes in nominal part geometry and in product structure, which are decisive on manufacturing and assembly costs. Additionally, more assembly technologies and methods will be analysed with a wider range of process planning related decisions, influencing both assembly operations and their sequences.

\section{Acknowledgements}

The authors would like to express their gratitude to all who supported the continuous scientific cooperation between the Hitachi Ltd. Research \& Development Group Japan, as CIRP Corporate Member, and the Institute of Computer Science and Control, Hungarian Academy of Sciences, started more than ten years ago. The Hungarian authors of the paper were partly supported by the Hungarian Scientific Research Fund (OTKA), Grant No. 113038.

\section{References}

[1] Hu SJ, Ko J, Weyand L, ElMaraghy HA, Lien TK, Koren Y, Bley H, Chryssolouris G, Nasr N, Shpitalni M (2011) Assembly System Design and Operations for Product Variety. CIRP Annals-Manufacturing Technology 60(2):715-735.

[2] Daaboul J Da Cunha C Bernard A, Laroche F (2011) Design for Mass Customization: Product Variety vs. Process Variety. CIRP AnnalsManufacturing Technology 60(1):169-174.

[3] Eric L, Fred H, Alain B, Emmanuel M, Corne S (2014) Tools and Techniques for Product Design. CIRP Annals-Manufacturing Technology 63(2): 607-630.

[4] Tolio T, Ceglarek D, ElMaraghy HA, Fischer A, Hu SJ, Lapierre L, Newman ST, Váncza J (2010) SPECIES-Co-evolution of products, processes and production systems, CIRP Annals-Manufacturing Technology 59(2):672-693.

[5] ElMaraghy H, Schuh G, ElMaraghy W, Piller F, Schönsleben P, Tseng M, Bernard A (2013) Product Variety Management. CIRP Annals-Manufacturing Technology 62(2): 629-652.

[6] Boothroyd G, Dewhurst P (1989) Product Design for Manufacture and Assembly. Boothroyd Dewhurst, Inc.,

[7] Miyakawa S, Ohashi T (1986) The Hitachi Assemblability Evaluation Method (AEM). Proceedings of the International Conference on Product Design for Assembly, Newport, Rhode Island, April 15-17.

[8] Eastman C (1996) Design for X, Concurrent Engineering Imperatives. Springer Netherlands.

[9] Redford A, Chal J (1994) Design for Assembly, Principle and Practice. McGrawHill Book Company Europe.

[10] Kretschmer R, Pfouga A, Rulhoff S, Stjepandić J (2017) Knowledge-based Design for Assembly in Agile Manufacturing by using Data Mining Methods. Advanced Engineering Informatics 33: 285-299.

[11] Ghandi S, Masehian E (2015) Review and Taxonomies of Assembly and Disassembly Path Planning Problems and Approaches. Computer-Aided Design 67-68:58-86.

[12] Xenia F, Iacopo $G$, Vei-chung $L$, Sudarsan $R$, Mahesh $M$, Conrad N, Xenia F, Iacopo G, Vei-chung L, Sudarsan R, Mahesh M, Conrad B, Carlos G, James T, (2007) An Ontology for Assembly Representation. NIST.

[13] He J (1991) Tolerancing for Manufacturing via Cost Minimization. International Journal of Machine Tools and Manufacture 31(4): 455-470.

[14] Andolfatto L, Thiébaut F, Lartigue C, Douilly M (2014) Quality- and Cost-driven Assembly Technique Selection and Geometrical Tolerance Allocation for Mechanical Structure Assembly. Journal of Manufacturing Systems 33(1):103115.

[15] Heling B, Aschenbrenner A, Walter M, Wartzack S (2016) On Connected Tolerances in Statistical Tolerance-Cost-Optimization of Assemblies with Interrelated Dimension Chains. Procedia CIRP 43:262-267. 